\title{
Simultaneous determination of lopinavir, saquinavir and ritonavirin in human plasma using liquid chromatography - ion trap mass spectrometry
}

\author{
Marcin Lipiński¹ ${ }^{\circledR}$, Krzysztof P. Bielawski², Ewa M. Słomińska³, \\ Ryszard T. Smoleński
}

\author{
${ }^{1}$ Department of Pharmaceutical Biochemistry, Medical University of Gdańsk, Poland \\ ${ }^{2}$ Laboratory of Molecular Diagnostics, Intercollegiate Faculty of Biotechnology, University of \\ Gdańsk and Medical University of Gdańsk, Poland \\ ${ }^{3}$ Department of Biochemistry, Medical University of Gdańsk, Poland
}

\begin{abstract}
Background: Lopinavir, saquinavir, and ritonavir are viral protease inhibitors (PIs) developed for and widely used in the therapy of human immunodeficiency virus (HIV)-related disease. These compounds are also active in vitro against the pathogens causing tuberculosis, malaria and coronavirus infections. Pls have been regarded as a platform for the design of inhibitors targeting severe acute respiratory syndrome coronavirus 2 (SARS-CoV-2)-encoded proteases. This study aimed to develop a liquid chromatography/mass spectrometry (LC/MS) procedure for accurate simultaneous determination of concentrations of these three PIs in the plasma. Methods: Samples of human plasma were protein precipitated with 0.3 $M$ zinc sulfate in a water/methanol solution (30:70, v/v). The extracts were analyzed with reversed-phase chromatography coupled with the electrospray ionization (ESI) source of the ion trap mass detector operating in mEass spectrometry (MS) and tandem mass spectrometry (MS/MS) modes. Results: Calibration curves demonstrated good linearity from 0.01 to $10 \mu \mathrm{g} / \mathrm{mL}$ and acceptable reproducibilities and recoveries. Conclusions: The described procedure proves that a very basic ion-trap LC/MS system could be applied for selective, rapid, and precise determination of antiviral protease inhibitors.
\end{abstract}

Keywords: antiviral therapy $\cdot$ lopinavir $\cdot$ saquinavir $\cdot$ ritonavir $\cdot$ LC/MS

\section{Citation}

Lipiński M, Bielawski KP, Słomińska EM, Smoleński RT. Simultaneous determination of lopinavir, saquinavir and ritonavir in human plasma using liquid chromatography - ion trap mass spectrometry. Eur J TransI Clin Med. 2021;4(2):60-67.

DOI: $10.31373 / \mathrm{ejtcm} / 139693$ 


\section{Abbreviations}

- ESI - electrospray ionization,

- HAART - highly active antiretroviral therapy,

- HIV - human immunodeficiency virus,

- LC/MS - liquid chromatography/mass spectrometry,

- MS/MS - tandem mass spectrometry,

- Pls - protease inhibitors,

- SARS-CoV-2 - severe acute respiratory syndrome coronavirus 2,

- TMD - therapeutic drug monitoring

\section{Introduction}

Lopinavir, saquinavir, and ritonavir are peptidomimetic inhibitors of human immunodeficiency (HIV) protease and belong to the protease inhibitors (PIs) group of drugs. They were developed to treat HIV/AIDS (acquired immunodeficiency syndrome) and are currently used in clinical practice in combinations with nucleoside inhibitors of HIV reverse transcriptase in the highly active antiretroviral therapy (HAART)[1-2]. Lopinavir and saquinavir (or other new generation PIs) are routinely combined with ritonavir in the so-called boosted protease inhibitor regimens because ritonavir administered in low doses can additionally block the cytochrome P450 (CYP3A4), thus increasing the bioavailability of the two first compounds [3].

In addition to its anti-retroviral properties, Pls exert anti-malarial activity targeting aspartyl proteases in the Plasmodium species and may be associated with a reduced incidence of malaria in HAART receivers [4-5]. Saquinavir has also appeared as a potential agent for tuberculosis, based on an in vitro study of a Mycobacterium tuberculosis infection model [6].

The policy of repurposing existing drugs has brought the three PIs to light as strong candidates for anti-coronavirus disease 2019 (COVID-19) drugs, specifically as potential inhibitors of severe acute respiratory syndrome coronavirus 2 (SARS-CoV-2)-encoded proteases. Saquinavir could block the proteolytic activity of SARS-CoV-2 3CLpro protease in vitro and the in silico predictions demonstrated its ability to bind the dimeric SARS-CoV-2 Mpro protease [7-8]. Lopinavir/ritonavir was previously demonstrated to have in vitro activity against both severe acquired respiratory syndrome coronavirus 1 (SARS-CoV) and Middle East respiratory syndrome coronavirus (MERS-CoV), with some efficacy in animal and clinical studies [9-10]. Despite the poor performance of PIs, when compared to the standard of care, in clinical studies with COVID-19 patients, these drugs can be still regarded as a platform to design new and more potent inhibitors of SARS-CoV proteases [8, 11-12].

In addition, the clinical data from HIV-infected patients indicate individual variations in responses to treatment, with some non-responders that demonstrate no pheno- type- or genotype-related resistance [3]. This insufficient response could result from specific pharmacokinetics in the individual subjects and the extent to which the patients follow the prescribed therapy. Information about the drug kinetics profiles of patients can be very important in clinical practice $[3,13]$. An optimal way to address it is to evaluate the Pls concentrations in the patient's plasma and to perform therapeutic drug monitoring. This study aimed to develop a liquid chromatography/mass spectrometry (LC) MS) procedure for accurate simultaneous determination of concentrations of these three PIs in the plasma.

Materials and methods

\section{Preparation of standards and a calibration curve}

The standards of lopinavir, saquinavir, and ritonavir (Fig. 1) were provided by Roche (Basel, Switzerland). Diazepam for use as an internal standard (IS) was from WZF Polfa (Warsaw, Poland). Stock solutions of drug standards were prepared at $1 \mathrm{mg} / \mathrm{mL}$ concentration by dissolving $5 \mathrm{mg}$ of ritonavir in

A

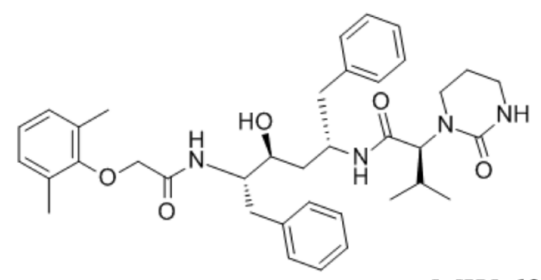

MW 628,81

B<smiles>CC(C)(C)NC(=O)C1CN(CC(O)[C@H](Cc2ccccc2)NC(=O)C(CC(N)=O)NC(=O)c2ccc3ccccc3n2)CC2(CCCCC2)C1</smiles>

MW 670,87<smiles>CC(C)c1nc(CN(C)C(=O)N[C@H](C(=O)NC(Cc2ccccc2)CC(O)C(Cc2ccccc2)NC(=O)OCc2cncs2)C(C)C)cs1</smiles>

MW 720,96

Figure 1. Chemical structures of three drugs: A. lopinavir, B. saquinavir, C. ritonavir. 
$5 \mathrm{~mL}$ of methanol, $5 \mathrm{mg}$ of saquinavir in $5 \mathrm{~mL}$ methanol/water $(50: 50, \mathrm{v} / \mathrm{v})$, and $5 \mathrm{mg}$ lopinavir in $5 \mathrm{~mL}$ of methanol/water $(50: 50, v / v)$. To assess the linearity of the assay, seven-point calibration curves were obtained by diluting the stock solutions in acetonitrile/water solution (AcN/W, 4:6, v/v), obtaining finally $0.01,0.03,0.1,0.3,1,3,10 \mu \mathrm{g} / \mathrm{mL}$ of each drug in $200 \mu \mathrm{l}$. Diazepam was added to the solutions as an internal standard (IS) at the final concentration of $5 \mu \mathrm{g} / \mathrm{mL}$.

\section{Plasma sample preparation}

0.3 M zinc sulfate (Sigma/Merck, Darmstadt, Germany) in water/methanol $(30: 70, \mathrm{v} / \mathrm{v})$ was used as an extracting solution for protein precipitation. Diazepam was added to the solutions on the day of extraction as an internal standard (IS) at the final concentration of $5 \mu \mathrm{g} / \mathrm{mL}$. To extract drugs from the plasma, $200 \mu \mathrm{L}$ of human plasma samples were mixed with $600 \mu \mathrm{l}$ of the extracting solution. Sample tubes were vortexed for $5 \mathrm{~min}$ and centrifuged at 13000 $\mathrm{rpm}$ for $3 \mathrm{~min}$. Supernatants were transferred into autosampler injection vials and analyzed with LC/MS.

\section{LC/MS analysis}

The system applied for the analysis was the LCQ Advantage ion trap mass detector equipped with the electrospray ionization (ESI) source connected to the Surveyor autosampler and the Surveyor quaternary gradient pump (Thermo-Finnigan, San Jose, USA). Separation was carried out on a BDS Hypersil $5 \mu \mathrm{m}$ column $(2 \mathrm{~mm} / 50 \mathrm{~mm}$ ) protected with a $2 \times 2 \mathrm{~mm}$ C18 Security Guard cartridge (Phenomenex, Torrance, USA). Buffer A was $10 \mathrm{mM}$ ammonium acetate, whereas buffer $B$ was acetonitrile. Starting conditions were $60 \% A / 40 \% B$ which changed linearly to $1 \% A / 99 \% B$ within $3 \mathrm{~min} .1 \% \mathrm{~A} / 99 \% \mathrm{~B}$ was maintained for $3 \mathrm{~min}$ and conditions returned to the initial values within the next $0.5 \mathrm{~min}$. The total injection cycle was $6 \mathrm{~min}$. The flow was maintained at $0.25 \mathrm{~mL} / \mathrm{min}$. The column temperature was maintained at $25^{\circ} \mathrm{C}$. The injection volume was $20 \mu \mathrm{L}$. The mass detector was operated in a positive ionization mode. Ion source parameters were optimized during direct drug infusion into the detector. The sheath gas flow rate was 35 arb. (instrument arbitrary units) and the spray voltage was $5.4 \mathrm{kV}$. The capillary temperature was set at $300^{\circ} \mathrm{C}$. Direct infusion allowed also to establish a fragmentation pattern of all three drugs and to optimize the collision energy for analysis in a fragmentation (tandem mass spectrometry, MS/MS) mode. Under those conditions, saquinavir formed a parent ion at $\mathrm{m} / \mathrm{z}=671.6$ with some sodium adduct at $\mathrm{m} / \mathrm{z}=693.6$, at quantities below $10 \%$. The isolated ion 671.6 when subjected to a collision energy of $45 \%$ resulted in the formation of a major fragment at $\mathrm{m} / \mathrm{z}=570.2$ which was used for detection. Ritonavir formed an ion at $\mathrm{m} / \mathrm{z}=721.3$ with about
$30 \%$ of sodium adduct $(\mathrm{m} / \mathrm{z}=743.3)$. Isolated ion 721.3 when subjected to a collision energy of $25 \%$ resulted in the formation of two major fragments at $\mathrm{m} / \mathrm{z}=295.1$ and 426.1 which were used for detection. Lopinavir formed a parent ion at $\mathrm{m} / \mathrm{z}=629.1$. The isolated ion 629.1 when subjected to a collision energy of $28 \%$ resulted in the formation of a major fragment at $\mathrm{m} / \mathrm{z}=447.1$ which was used for detection. During chromatographic separation, MS detector was set to operate in alternate full MS $(100-1000 \mathrm{~m} / \mathrm{z}) /$ selective ion or reaction monitoring modes ( $\mathrm{m} / \mathrm{z}: 286,671.6>570.2$ (45\%), $721.3>295.2 \& 426.1(25 \%)$, and $629.1>447.1(28 \%))$.

\section{Determination of reproducibility and recovery}

To determine reproducibility and recovery, we continued analyses on separate days with five injections of calibration standards and a quality control sample each day. Fresh samples were prepared each day. The data is presented in Table 1.

Table 1: Reproducibility and recovery data from patients plasma extracts analysis at three different concentrations of each drug (3, 0.3 , and $0.03 \mu \mathrm{g} / \mathrm{mL}$ ). Av: average, SD: standard deviation, CV: coefficient of variation, Rec: percentage of the recovery

\begin{tabular}{|c|c|c|c|}
\hline $3 \mathrm{mg} / \mathrm{L}$ & lopinavir & saquinavir & ritonavir \\
\hline Av & 288.2 & 295.7 & 280.1 \\
\hline SD & 16.79 & 15.32 & 24.35 \\
\hline CV & 0.058 & 0.051 & 0.086 \\
\hline Rec & $96 \%$ & $98 \%$ & $93 \%$ \\
\hline $0.3 \mathrm{mg} / \mathrm{mL}$ & lopinavir & saquinavir & ritonavir \\
\hline Av & 28.14 & 29.02 & 27.24 \\
\hline SD & 0.78 & 2.1 & 1.46 \\
\hline CV & 0.027 & 0.071 & 0.05 \\
\hline $\operatorname{Rec}$ & $93 \%$ & $96 \%$ & $90 \%$ \\
\hline $0.03 \mathrm{mg} / \mathrm{mL}$ & lopinavir & saquinavir & ritonavir \\
\hline Av & 1.78 & 2.15 & 2.22 \\
\hline SD & 0.45 & 0.32 & 0.59 \\
\hline $\mathrm{CV}$ & 0.25 & 0.14 & 0.26 \\
\hline Rec & $59 \%$ & $71 \%$ & $74 \%$ \\
\hline
\end{tabular}


We also used a negative control subject (treated with neither of the target compounds) to determine recovery at three different concentrations of each drug $(3,0.3$, and $0.03 \mu \mathrm{g} / \mathrm{mL})$.

\section{Results and Discussion}

As demonstrated in the chromatograms/LC reports in Figure 2, the internal standard (diazepam), lopinavir, saquinavir and ritonavir separated well chromatographically as standards, although with the possibility of construction of separate chromatograms for selected ions such a separation is not an absolute requirement. However, the problem with carryover occurred during several primary injections of the standards. We confirmed the presence of traces of analyzed compounds in compound-free samples following injections of higher concentrations of the standards. We solved this problem, using acetonitrile/water as a wash solution. The response of chromatographic peak areas for chromatograms extracted for selected ions was linear within the concentration range (between 0.01 and 10 $\mu \mathrm{g} / \mathrm{mL}$ ) for ritonavir, saquinavir, and lopinavir. Recoveries of drug standards added to the 4 plasma were $>90 \%$ (Table 1 ). The chromatograms/reports for the calibration curve are attached to demonstrate the signal-to-noise ratio (Fig. 2). Figure 3 represents chromatograms obtained in the analysis of the control plasma extract from an individual non-treated with the analyzed therapeutics, for comparison. Figure 4 with representative chromatograms/reports from the analyses of the treated patients' plasma extracts demonstrates that the analyzed drugs, if present, form clear peaks.

Our method, although applied for monitoring of just three compounds, can be suitable for monitoring other drugs in the same sample. Low sensitivity preliminary data could be obtained from a full MS trace of already recorded chromatograms, while more accurate determination would require extending the selective ion monitoring/fragmentation mode and re-run of the separation.

Several previously described analytical methods using high-performance liquid chromatography (HPLC) for evalu-

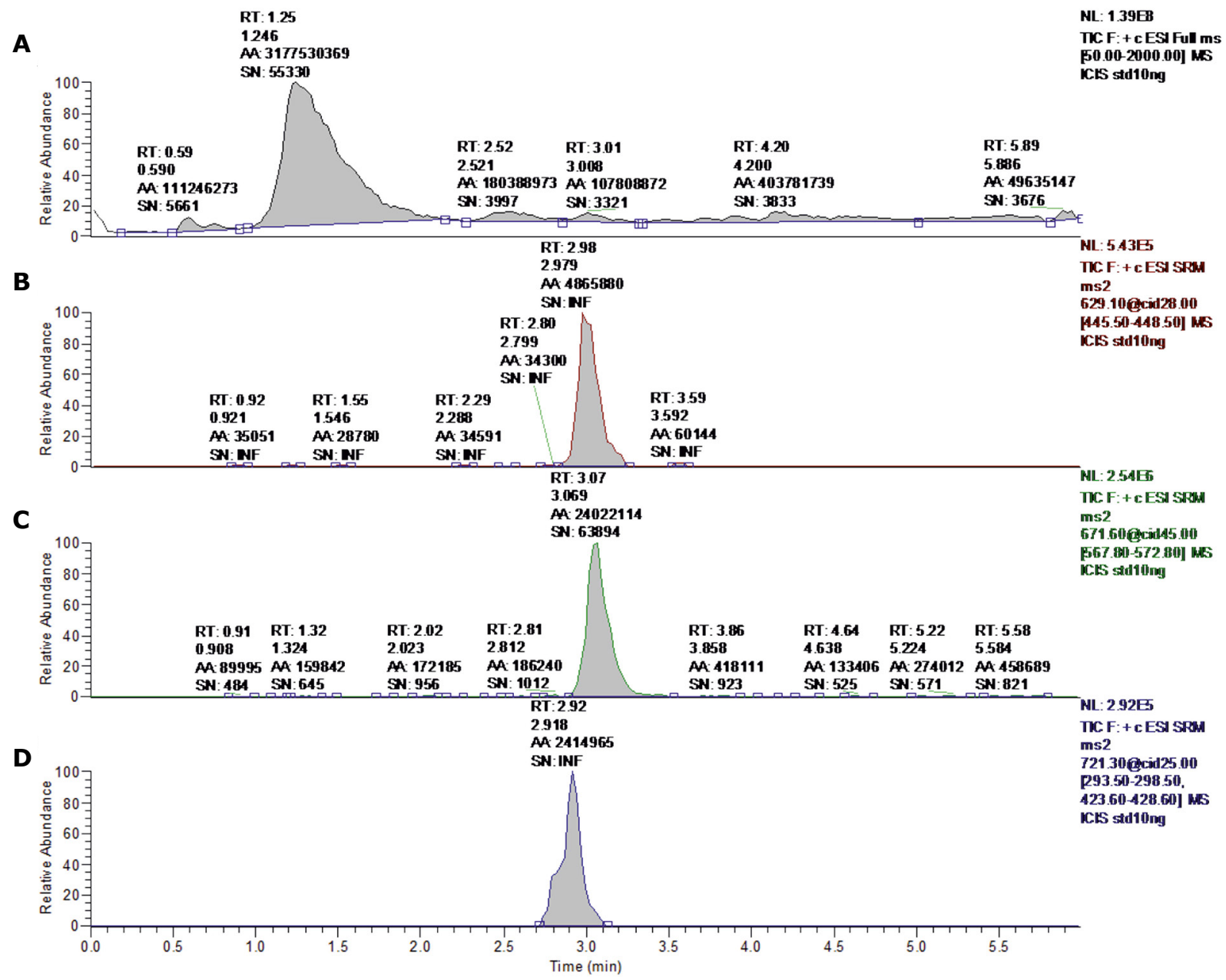

Figure 2. LC/MS chromatograms for standards of protease inhibitors in MS/MS mode: A. Total lon Current. B. lopinavir, C. saquinavir, D. ritonavir 
A
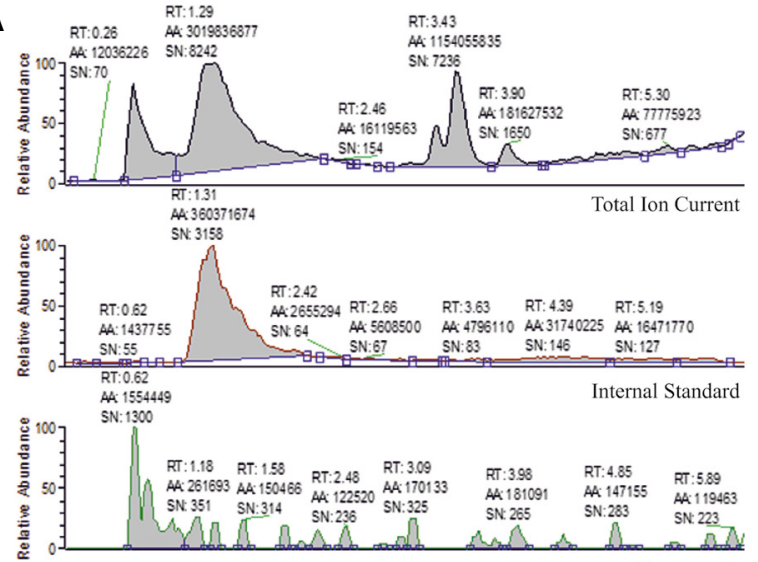

Full MS mode

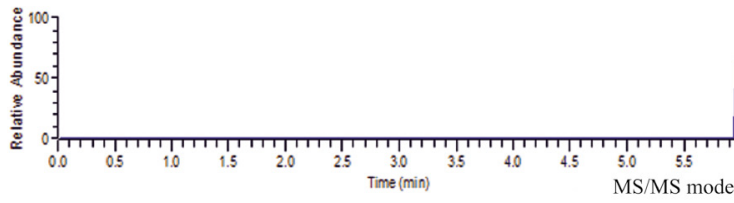

ation of the plasma levels of antiretroviral drugs have been established only for individual compounds [14] or for more than one, but with different extraction procedures and chromatographic conditions for each compound. That type of analytical procedure is expensive and time-consuming [15-17]. Some other methods used liquid chromatography but with different detection modes [18-20]. Our procedure seems to be much less expensive due to the implementation of the ion trap detector type which provides considerably lower operating costs than other mass detector types. While most of the procedures employ solid-phase extraction for material preparation, we used a simple precipitation procedure with methanol/zinc sulfate for the drug determination. Finally, the total run time of 6 minutes is significantly shorter than in many others assays. Then, the established method can be suitable for routine analyses of a large number of samples in a very short time.

Although viral protease inhibitors described in this study take their origin from HIV treatment, their future use in the therapies of other diseases (e.g. malaria) is possible. There is still an urgent need for repurposed or new anti-SARS-CoV-2 therapeutics with sufficient clinical outcomes. So far four COVID-19 vaccines have been conditionally approved by the European Medicines Agency: 2 are based on the mRNA technology (Moderna and Pfizer/BioNTech) and 2 use the adenovirus platforms (AstraZeneca and Janssen/Johnson and Johnson) [21] and the number of vaccinated individuals has been increasing. However, fast-acting and efficient antiviral therapy based on the clinically well-defined compounds seems to be a necessary approach for the still recorded severely-ill COVID-19 patients, particularly in the parts of the world where the progress in vaccination is limited. The plasma drug concentrations of lopinavir/ritonavir administered in
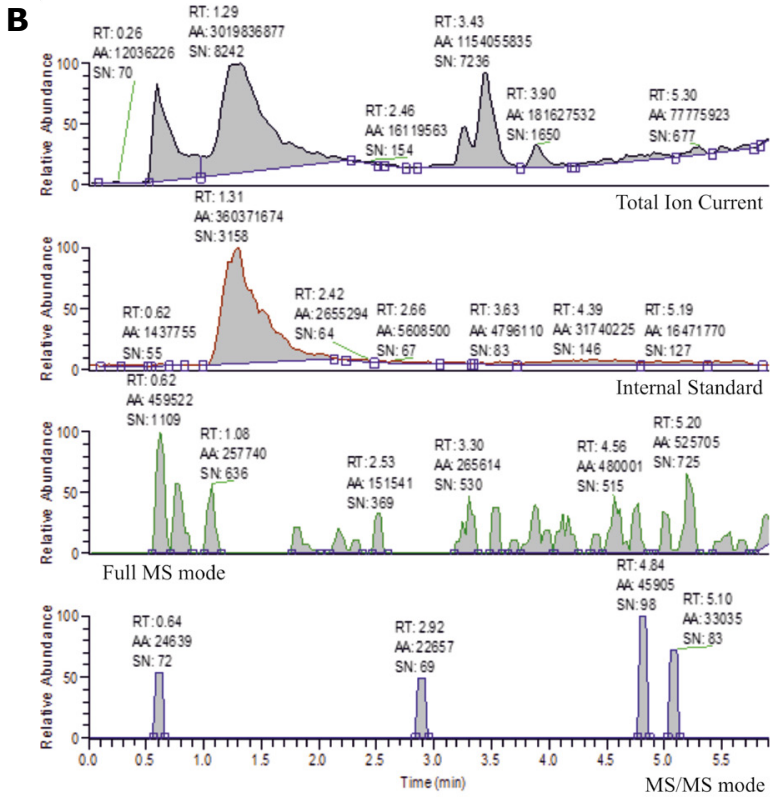

C
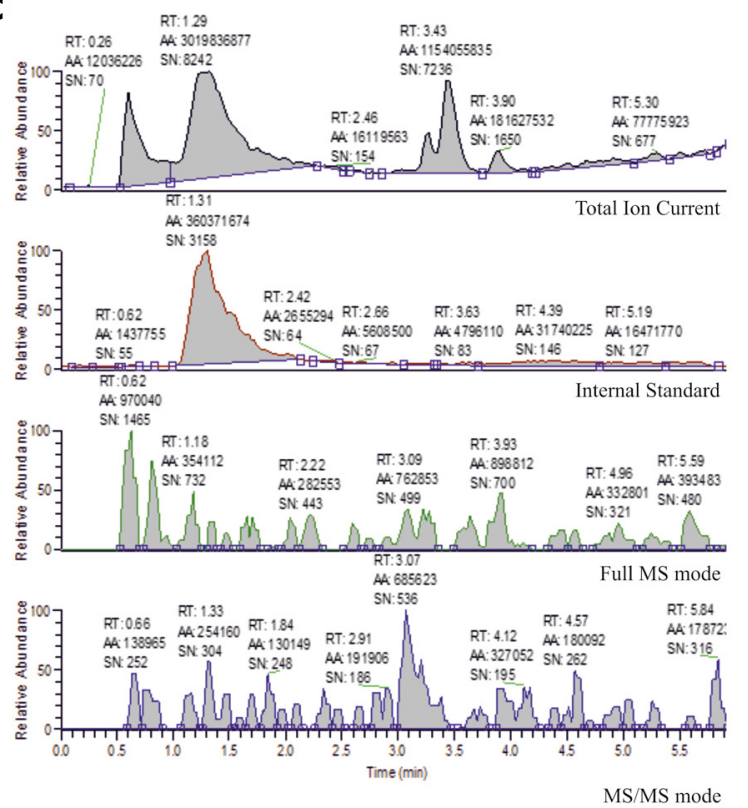

Figure 3. LC/MS chromatograms for the plasma extract of a control subject. Ion chromatograms for: A. lopinavir, B. saquinavir, and C. ritonavir. Each chromatogram consists of: Total lon Current, Internal standard, Full MS mode, and MS/MS mode

typical doses do not reach the levels that may be needed to inhibit SARS-CoV-2 proteases [22] and their use for COVID-19 treatment is currently not recommended. However, the data predicting saquinavir activity have appeared only recently [78], therefore if this drug is used in clinical tests, a method to control the concentration of this drug in the plasma with an inexpensive LC/MS system could be very useful for optimization and monitoring of such treatment. 
A
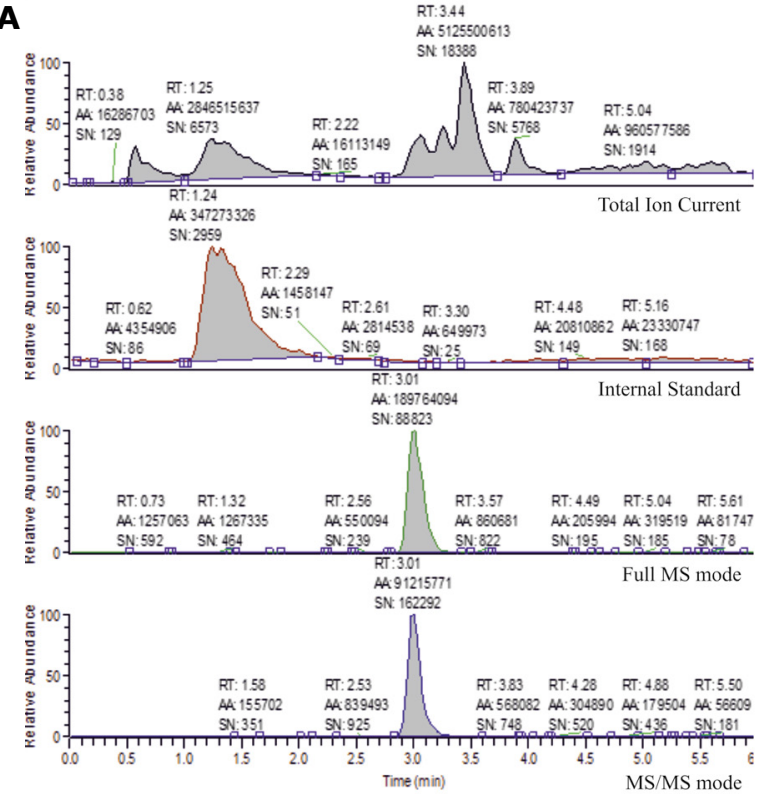

\section{Conclusions}

We developed an LC/MS procedure employing basic and most commonly accessible ion-trap LC/MS system and demonstrated that it could be used for simultaneous selective, rapid, and precise determination of the plasma concentrations three antiviral PIs. The described method can be adapted for high-throughput analyses in routine therapeutic drug monitoring.

\section{Acknowledgments}

The experiments conducted in this study were funded by the Polish National Science Centre (NCN) Harmonia grant no: 2016/22/M/NZ4/00678.

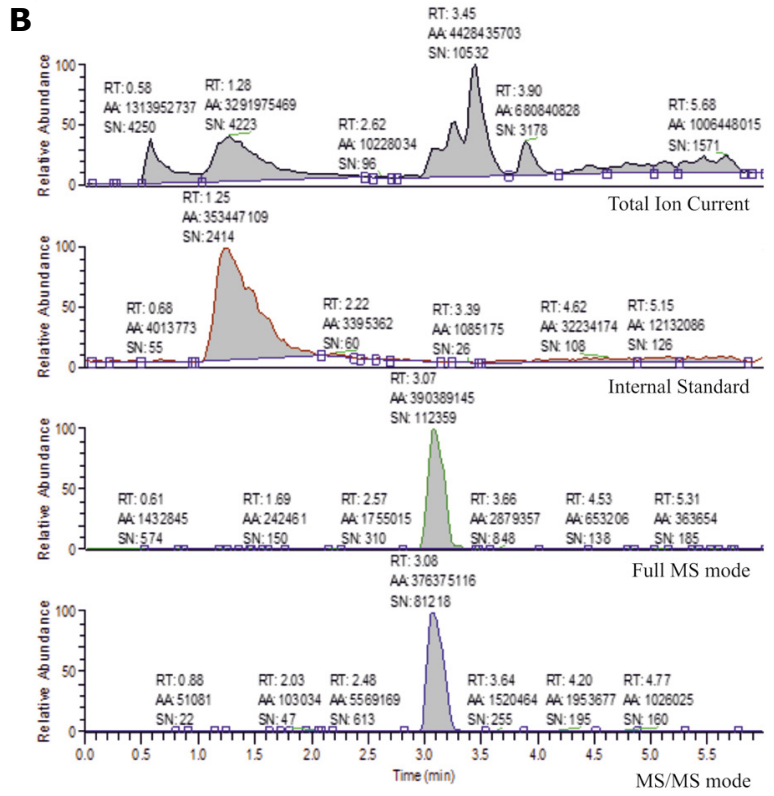

C
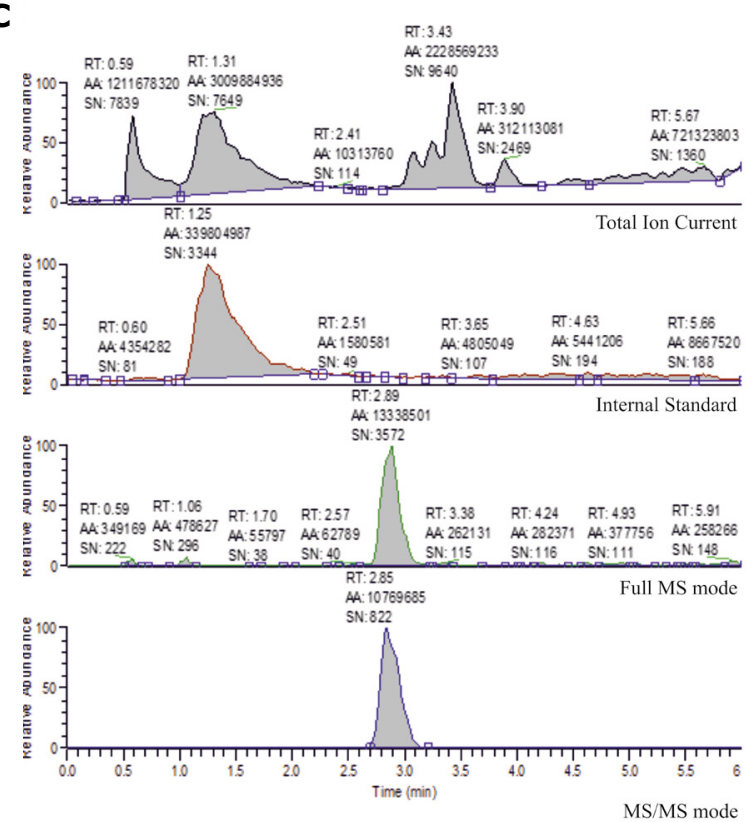

Figure 4. LC/MS chromatograms for plasma extract from a patient treated with: A. lopinavir, B. saquinavir, and C. ritonavir. Each chromatogram consists of: Total lon Current, Internal standard, Full MS mode, and MS/MS mode

\section{References}

1. De Clercq E. New developments in anti-HIV chemotherapy. Biochim Biophys Acta - Mol Basis Dis [Internet]. 2002 Jul;1587(2-3):258-75. Available from: https://www.sciencedirect.com/science/article/pii/S0925443902000893

2. De Clercq E. Antiviral drugs in current clinical use. J Clin Virol [Internet]. 2004 Jun;30(2):115-33. Available from: https:// www.sciencedirect.com/science/article/pii/S1386653204000459 
3. King JR, Wynn H, Brundage R, Acosta EP. Pharmacokinetic Enhancement of Protease Inhibitor Therapy. Clin Pharmacokinet [Internet]. 2004;43(5):291-310. Available from: https://doi.org/10.2165/00003088-200443050-00003

4. Nsanzabana C, Rosenthal PJ. In Vitro Activity of Antiretroviral Drugs against Plasmodium falciparum. Antimicrob Agents Chemother [Internet]. 2011 Nov;55(11):5073-7. Available from: https://journals.asm.org/doi/10.1128/AAC.05130-11

5. Achan J, Kakuru A, Ikilezi G, Ruel T, Clark TD, Nsanzabana C, et al. Antiretroviral Agents and Prevention of Malaria in HIV-Infected Ugandan Children. N Engl J Med [Internet]. 2012 Nov 29;367(22):2110-8. Available from: https://doi. org/10.1056/NEJMoa1200501

6. Pires D, Valente S, Calado M, Mandal M, Azevedo-Pereira JM, Anes E. Repurposing Saquinavir for Host-Directed Therapy to Control Mycobacterium Tuberculosis Infection. Front Immunol [Internet]. 2021 Mar 26;12:890. Available from: https://www.frontiersin.org/article/10.3389/fimmu.2021.647728

7. Chiou W-C, Hsu M-S, Chen Y-T, Yang J-M, Tsay Y-G, Huang H-C, et al. Repurposing existing drugs: identification of SARSCoV-2 3C-like protease inhibitors. J Enzyme Inhib Med Chem [Internet]. 2021 Jan 1;36(1):147-53. Available from: https:// doi.org/10.1080/14756366.2020.1850710

8. Luo S, Huang K, Zhao X, Cong Y, Zhang JZH, Duan L. Inhibition mechanism and hot-spot prediction of nine potential drugs for SARS-CoV-2 M pro by large-scale molecular dynamic simulations combined with accurate binding free energy calculations. Nanoscale [Internet]. 2021;13(17):8313-32. Available from: http://xlink.rsc.org/?DOI=D0NR07833F

9. Chu CM. Role of lopinavir/ritonavir in the treatment of SARS: initial virological and clinical findings. Thorax [Internet]. 2004 Mar 1;59(3):252-6. Available from: http://thorax.bmj.com/content/59/3/252.abstract

10. Arabi YM, Alothman A, Balkhy HH, Al-Dawood A, AlJohani S, Al Harbi S, et al. Treatment of Middle East Respiratory Syndrome with a combination of lopinavir-ritonavir and interferon- $\beta 1 \mathrm{~b}$ (MIRACLE trial): study protocol for a randomized controlled trial. Trials [Internet]. 2018 Dec 30;19(1):81. Available from: https://doi.org/10.1186/s13063-017-2427-0

11. Lecronier M, Beurton A, Burrel S, Haudebourg L, Deleris R, Le Marec J, et al. Comparison of hydroxychloroquine, lopina$\mathrm{vir} /$ ritonavir, and standard of care in critically ill patients with SARS-CoV-2 pneumonia: an opportunistic retrospective analysis. Crit Care [Internet]. 2020 Dec 11;24(1):418. Available from: https://doi.org/10.1186/s13054-020-03117-9

12. Flisiak R, Zarębska-Michaluk D, Berkan-Kawińska A, Tudrujek-Zdunek M, Rogalska M, Piekarska A, et al. Remdesivir-based therapy improved recovery of patients with COVID-19 in the SARSTer multicentre, real-world study. medRxiv [Internet]. 2020 Jan 1;2020.10.30.20215301. Available from: http://medrxiv.org/content/early/2020/11/03/2020.10.30.20215301.abstract

13. la Porte CJL, Wasmuth J-C, Schneider K, Rockstroh JK, Burger DM. Lopinavir/ritonavir plus saquinavir in salvage therapy; pharmacokinetics, tolerability and efficacy. AIDS [Internet]. 2003;17(11):1700-2. Available from: https://journals.Iww. com/aidsonline/Fulltext/2003/07250/Lopinavir ritonavir plus saquinavir in salvage.18.aspx

14. Colombo S, Guignard N, Marzolini C, Telenti A, Biollaz J, Decosterd LA. Determination of the new HIV-protease inhibitor atazanavir by liquid chromatography after solid-phase extraction. J Chromatogr B [Internet]. 2004 Oct;810(1):25-34. Available from: https://www.sciencedirect.com/science/article/pii/S157002320400580X

15. Rouzes A, Berthoin K, Xuereb F, Djabarouti S, Pellegrin I, Pellegrin J, et al. Simultaneous determination of the antiretroviral agents: amprenavir, lopinavir, ritonavir, saquinavir and efavirenz in human peripheral blood mononuclear cells by high-performance liquid chromatography-mass spectrometry. J Chromatogr B [Internet]. 2004 Dec 25;813(1-2):209-16. Available from: https://www.sciencedirect.com/science/article/pii/S1570023204007779

16. Takahashi M, Yoshida M, Oki T, Okumura N, Suzuki T, Kaneda T. Conventional HPLC Method Used for Simultaneous Determination of the Seven HIV Protease Inhibitors and Nonnucleoside Reverse Transcription Inhibitor Efavirenz in Human Plasma. Biol Pharm Bull [Internet]. 2005;28(7):1286-90. Available from: http://www.jstage.jst.go.jp/article/ $\underline{\mathrm{bpb} / 28 / 7 / 28 \quad 71286 / \text { article }}$

17. Droste JAH, Verweij-van Wissen CPWGM, Burger DM. Simultaneous Determination of the HIV Drugs Indinavir, Amprenavir, Saquinavir, Ritonavir, Lopinavir, Nelfinavir, the Nelfinavir Hydroxymetabolite M8, and Nevirapine in Human Plasma by Reversed-Phase High-Performance Liquid Chromatography. Ther Drug Monit [Internet]. 2003;25(3). Available from: https://journals.Iww.com/drug-monitoring/Fulltext/2003/06000/Simultaneous Determination of the HIV Drugs.23.aspx

18. Titier K, Lagrange F, Péhourcq F, Edno-Mcheik L, Moore N, Molimard M. High-Performance Liquid Chromatographic Method for the Simultaneous Determination of the Six HIV-Protease Inhibitors and Two Non-Nucleoside Reverse Transcriptase Inhibitors in Human Plasma. Ther Drug Monit [Internet]. 2002;24(3). Available from: https://journals.Iww.com/ drug-monitoring/Fulltext/2002/06000/High Performance Liquid Chromatographic Method for.15.aspx

19. Keil K, Frerichs VA, DiFrancesco R, Morse G. Reverse Phase High-Performance Liquid Chromatography Method for the Analysis of Amprenavir, Efavirenz, Indinavir, Lopinavir, Nelfinavir and Its Active Metabolite (M8), Ritonavir, and Saquina- 
vir in Heparinized Human Plasma. Ther Drug Monit [Internet]. 2003;25(3). Available from: https://journals.Iww.com/ drug-monitoring/Fulltext/2003/06000/Reverse Phase High Performance Liquid.15.aspx

20. van Heeswijk RP., Hoetelmans RM., Harms R, Meenhorst P., Mulder J., Lange JM., et al. Simultaneous quantitative determination of the HIV protease inhibitors amprenavir, indinavir, nelfinavir, ritonavir and saquinavir in human plasma by ion-pair high-performance liquid chromatography with ultraviolet detection. J Chromatogr B Biomed Sci Appl [Internet]. 1998 Nov;719(1-2):159-68. Available from: https://www.sciencedirect.com/science/article/pii/S0378434798003922

21. Creech CB, Walker SC, Samuels RJ. SARS-CoV-2 Vaccines. JAMA [Internet]. 2021 Apr 6;325(13):1318-20. Available from: https://doi.org/10.1001/jama.2021.3199

22. Schoergenhofer C, Jilma B, Stimpfl T, Karolyi M, Zoufaly A. Pharmacokinetics of lopinavir and ritonavir in patients hospitalized with coronavirus disease 2019 (COVID-19). Ann Intern Med [Internet]. 2020 Oct 20;173(8):670-2. Available from: https://www.acpjournals.org/doi/10.7326/M20-1550 